\title{
Efficacy of Rose Oil Soft Capsules on Clinical Outcomes in Ulcerative Colitis: A Pilot Randomized, Double-Blinded, Placebo-Controlled Clinical Trial
}

\author{
Ali Tavakoli ${ }^{1,2}$, Meysam Shirzad ${ }^{3}$, Alireza Taghavi ${ }^{4}$, Mohammadreza Fattahi ${ }^{4}$, Mohammadmahdi Ahmadian-Attari ${ }^{5}$, \\ Leila Mohammad Taghizadeh ${ }^{6}$, Mahsa Rostami Chaijan ${ }^{1,2}$, Masih Sedigh Rahimabadi ${ }^{7,8}$, Rahimeh Akrami ${ }^{1,2}$, Mehdi Pasalar $^{1,8} \bowtie$
}

\footnotetext{
${ }^{1}$ Research Center for Traditional Medicine and History of Medicine, Shiraz University of Medical Sciences, Shiraz, Iran ${ }^{2}$ Department of Traditional Medicine, Faculty of Medicine, Shiraz University of Medical Sciences, Shiraz, Iran

${ }^{3}$ Department of Persian Medicine, School of Persian Medicine, Tehran University of Medical Sciences, Tehran, Iran

${ }^{4}$ Gastroenterohepatology Research Center, Shiraz University of Medical Sciences, Shiraz, Iran

${ }^{5}$ Evidence-based Phytotherapy and Complementary Medicine Research Center, Alborz University of Medical Sciences, Karaj, Iran

${ }^{6}$ Department of Traditional Medicine, Medicinal Plants Research Center of Barij, Kashan, Iran

${ }^{7}$ Department of Persian Medicine, Fasa University of Medical Sciences, Fasa, Iran

${ }^{8}$ Essence of Parsiyan Wisdom Institute, Traditional Medicine and Medicinal Plant Incubator, Shiraz University of Medical Sciences, Shiraz, Iran
}

\begin{abstract}
Background: Ulcerative colitis is the most common form of inflammatory bowel disease worldwide, which presents with superficial ulcers in the rectum and colon. The aim of this study was to assess the effectiveness of rose oil soft capsules over placebo on the clinical outcomes in moderate to severe ulcerative colitis. Materials and Methods: This study was a pilot randomized, double-blind clinical trial, and the 40 patients were assigned into rose oil and placebo groups ( $\mathrm{n}=20$ per group). All patients were instructed to use their prescribed two soft capsules three times daily for two months. The clinical symptoms, quality of life the patients, and calprotectin level were evaluated via partial Mayo clinic score, irritable bowel disease questionnaire (IBDQ-9), and calprotectin kit as primary outcome measures. Results: The mean age of the participants was $41 \pm 10$ years. Most of them (53.6\%) were male, and the remaining $(46.4 \%)$ were female. The demographic and baseline data showed no differences between the two groups. Partial Mayo clinic scores decreased in both groups after the treatment, but the difference between the rose oil and placebo groups was not statistically significant $(\mathrm{P}=0.99)$. IBDQ-9 score also increased in both interventions before and after the treatment $(\mathrm{P}=0.012)$, though the differences between these two groups were not statistically significant $(\mathrm{P}=0.61)$. There were no significant differences between the two study groups either in terms of calprotectin level $(\mathrm{P}=0.219)$. Conclusion: This study showed that rose oil might improve ulcerative colitis clinical outcomes, but for a better evaluation, it is imperative to conduct experiments with a large sample size and longer follow-up observation. [GMJ.2019;8:e1307] DOI:10.31661/gmj.v8i0.1307
\end{abstract}

Keywords: Ulcerative Colitis; Rose Oil; Clinical Trial; Calprotectin

\section{GMJ}

Copyright(C) 2019, Galen Medical Journal. This is an open-access article distributed under the terms of the Creative Commons Attribution 4.0 International License (http://creativecommons.org/licenses/bv/4.0/) Email:info@gmj.ir

\author{
Correspondence to: \\ Mehdi Pasalar, Research Center for Traditional Medi- \\ cine and History of Medicine, Shiraz University of Med- \\ ical Sciences, Shiraz, Iran \\ Telephone Number: 07132338476 \\ Email Address: pasalar@sums.ac.ir
}




\section{Introduction}

U lcerative colitis (UC) is a chronic, relapsing-remitting inflammatory disease of the intestine. It is the most common form of inflammatory bowel disease (IBD) worldwide characterized by inflammatory changes and superficial ulcerations in the rectum and colon $[1,2]$. UC has distinct pathological and clinical features, but its etiology and exact pathogenesis are unknown. Symptoms of this disease can include increased defecation frequency, rectal bleeding, urgency or tenesmus, abdominal cramps and pain, depending on the extent and severity of the disease. UC can seriously impact the health-related quality of life (HRQoL) of patients and has significant social, psychological, and financial repercussions $[3,4]$. UC treatment aims to induce and maintain clinical remission, improve HRQoL, and mucosal healing $[1,5]$. The first-line therapy involves 5-aminosalicylate (5-ASA) compounds - which have been successful in approximately $50 \%$ of patients - and next line therapy includes steroids, immunosuppressants, and biological drugs that have risks of infection and malignancy $[2,4,6]$. Therefore, it is imperative to explore new and safe remedies with a natural origin as a complementary/ alternative therapy for this disease [7].

Rose oil is one of these remedies, which is prepared by soaking the whole petals of damask rose (Rosa damascena) in sesame oil as the carrier. In traditional manuscripts, rose oil with this method of preparation has been considered as an anti-inflammatory agent especially in the gastrointestinal tract $[8,9]$. The $R$. damascena Mill L. -commonly known as damask rose - is known as Gol-e Mohammadi in Iran. It is one of the most important species of the Rosaceae family [10]. R. damascena has traditionally been used as a hypnotic, cough suppressant, anti-inflammatory, anti-ulcer, gentle laxative agent, and for treating digestive problems $[11,12]$. At present, in addition to its perfuming effects, flowers, petals and hips (seed-pot) of this plant are also used for medical purposes. New studies have proven the effectiveness of the components of damask rose and rose oil in animal models of IBD and inflammation [13, 14]. UC is a recurrent and refractory disease that has no permanent cure, so it can result in significant long-term morbidity, physical and psychological discomfort, thereby affecting the quality of life (QoL) of patients [1]. Furthermore, its treatment is expensive (especially biological drugs), and medications may have serious side effects [15]. Accordingly, the discovery of novel complementary/alternative drugs including those from natural sources that overcome the abovementioned drawbacks of the current therapy is of great interest [16]. The aim of this study was to evaluate the efficacy of rose oil in comparison with placebo in controlling symptoms of UC.

\section{Materials and Methods}

\section{Patients}

The patients were chosen from those who had a clinical diagnosis of UC based on colonoscopy and allied pathology report. They were visited by a gastroenterologist with more than 15 years of experience at the beginning of the trial.

\section{Sample Size Calculation}

Considering alpha $=0.05$, the power of study equal to $80 \%$, and mean of partial Mayo clinic score of 4 with a standard deviation of 2 , we hypothesized that our intervention would show a $25 \%$ accepted effect size. Thus, the sample size was determined as 20 patients in each group with 1:1 allocation ratio.

\section{Inclusion and Exclusion Criteria}

Patients with definite diagnosis of UC who showed moderate to severe disease based on the diagnostic criteria (classified using the partial Mayo clinic score as reference) and previously confirmed by colonoscopy were recruited in the trial. All patients were above 18 years old, and there were no limitations on gender. All patients volunteered to sign an informed consent. Exclusion criteria included as follow; 1. Patients who refused to provide a signed informed consent for participating in the study; 2. Patients with a history of using steroids for other diseases within four weeks before entering the current study; 3. Patients with a history of consuming any non-steroidal anti-inflammatory drugs within one week be- 
fore entering the study; 4. Patients with a history of antibiotic consumption or any medicinal plants within two weeks before entering the study; 5. Patients with a positive history of allergy or hypersensitivity to any component of the rose oil or herbal medicine; 6. Patients who were in the lactation period or pregnant. The patients whose symptoms worsened, those with serious adverse reactions during treatment, or patients who voluntarily quit or were found to be ineligible for the study by the investigators were also excluded from the study. If the patient did not pass the treatment period, they would be excluded from the study. Other exclusion cases were due to new positive pregnancy test and personal request due to health considerations.

\section{Randomization, Blinding, and Allocation Concealment}

A package of medication had been considered for each patient with a numeric label containing soft capsules for consumption for two months. The medications for both groups were produced by Barij Essence Pharmaceutical Co. (Iran) and also randomly allocated based on a randomized list. They were completely the same in terms of color, shape, and packaging. Concealment was done by a research assistant who was not aware of the study protocol. The randomized list was generated using Microsoft Excel 2010, USA with a block randomization method. Accordingly, 40 eligible participants were also assigned randomly into two parallel groups with a 1:1 allocation ratio. The participants, gastroenterologist, medical researcher assistant, and statistician were blinded to the allocation of the patients.

\section{Treatment Protocol}

Treatments consisted of a soft capsule 1000 $\mathrm{mg}$ to be taken orally. One group received rose oil soft capsule, and the other received placebo soft capsule filled with liquid paraffin. Both soft capsules were the product of Barij Essence Pharmaceutical Company. Patients in both groups were given two capsules three times daily half an hour before meal. The treatment duration for both groups was two months. During the study, the patients were allowed to take concomitant or current med- ications for UC. The patients were followed for two months and visited three times, once on arrival and twice at the end of each month. The questionnaires about patients' demographic data and disease course were filled out at the baseline visit. Clinical symptoms and QoL of the patients were evaluated by partial Mayo clinic standard form and Persian version of IBD questionnaire-9 (IBDQ-9) [18]. The partial Mayo clinic standard form was filled at the first visit and then at the end of each month by the medical research assistant. Also, the IBDQ-9 form was completed at the beginning of the study and after two months of treatment by the medical research assistant. By the final visit, the questionnaires about patient's satisfaction and ease of usage were filled out. Any adverse events, recorded in a diary, were collected by a medical research assistant at the last appointment. All the history takings were done by the medical research assistant, and all the participants were examined by one expert gastroenterologist.

\section{Primary Outcomes}

The primary outcome measures included: 1 . Clinical symptoms before and after treatment, as evaluated by partial mayo clinic score [17]; 2. QoL scores before and after the treatment, as evaluated by IBDQ-9 with proven validity and reliability in previous studies; IBDQ-9 is a specific QoL questionnaire in IBD patients, which is under usage in more than 10 languages. It contains the following domains: bowel symptoms, emotional status, systemic symptoms, and social function [18]. 3. Calprotectin levels before and after treatment assessed by fecal calprotectin using a commercially available enzyme-linked immunosorbent assay (Buhlmann Laboratories AG, Schonenbuch, Switzerland) [19].

\section{Data Collection}

This study was a single-center, 2-arm, randomized, double-blind, placebo-controlled clinical trial conducted in Motahari Clinic of Shiraz University of Medical Sciences, gastroenterology clinic, the main academic center of southern Iran, from October 2016 to November 2017. The data collection was conducted in this period according to the trial design. 


\section{Secondary Outcomes}

The secondary outcome measures involved evaluating the patients' satisfaction and the number of participants with any adverse events. The patients' satisfaction was assessed by a visual analog scale, ranging from 1 (least satisfactory) to 10 (most satisfactory). The adverse events were recorded on a diary during the treatment time.

\section{Ethical Issues}

The current study was registered at the Iranian Registry of Clinical Trials (IRCT registration number: IRCT2016120323823N2). All participants provided a signed informed consent form before the trial. Furthermore, the Ethics Committee of Shiraz University of Medical Sciences approved the protocol for this study (reference number: IR.SUMS. REC.1394.211), and there were no significant changes during the experiment.

\section{Statistical Analysis}

The data were analyzed via the SPSS software (IBM Corp, Released 2013, IBM SPSS Statistics for Windows, version 22.0 Armonk, NY). The frequency (percent), mean \pm standard deviation (SD) and median were used for the data description. Independent sample $t$-test, Mann-Whitney, and Chi-Square test were used for comparing the difference between the two groups. Wilcoxon signed-rank test and paired sample $t$-test were applied to compare the difference within an intervention group. Analysis of covariance (ANCOVA) was also used for comparing both groups adjusted for the baseline variables. The differences were considered statistically significant when the $\mathrm{P}$-value was $<0.05$.

\section{Results}

A total of 40 patients were divided into two groups: 20 patients in the rose group and 20 patients in the placebo group. Finally, 28 patients (14 in the rose oil group and 14 in the placebo group) completed the study. Twelve participants $(30 \%)$ withdrew from the study; 9 of them did not come for the second visit (5 in the rose group and 4 in the placebo group), and 3 patients did not come for the third visit (Figure-1). All patients completed the baseline screening. There was no statistically significant difference between these two groups in terms of baseline demographic characteristics (age and gender), disease course (duration, number of admissions, extent of the disease), clinical symptoms (defecation frequency and rectal bleeding), partial Mayo clinic score, and IBDQ-9 $(\mathrm{P}>0.05)$ at baseline visits. It suggests that the baseline data for the groups were balanced and comparable (Table-1). Concomitant medication was also similar in both study groups without statistically significant difference $(\mathrm{P}=0.77)$. Partial Mayo clinic score significantly decreased in both groups after the treatment $(\mathrm{P}<0.05)$, but the differences between these two groups were not statistically significant ( $P>0.05$, Table-2). IBDQ-9 revealed a significant improvement after the treatment compared with the baseline levels in both groups $(\mathrm{P}<0.05)$, but the differences between these two groups were not statistically significant $(\mathrm{P}>0.05$, Table-2). Mean of fecal calprotectin levels decreased in both groups at the end of the study; however, this difference was not statistically significant (Table-3). The between-group analysis also showed no significant difference after the trial $(\mathrm{P}=0.219)$. Although there was greater patients' satisfaction in the rose group $(7.46 \pm 2.33$ vs. $6.79 \pm$ 2.01 ), the statistical analyses showed no significant difference between these groups ( $\mathrm{P}$ $=0.426$, Table-4). Among 20 patients treated with rose oil, $2(10 \%)$ withdrew from treatment because of gastrointestinal adverse events, as compared with 3 of 20 patients receiving liquid paraffin $(15 \%)$. The statistical analysis indicated no significant difference between the two study groups in terms of side effects $(\mathrm{P}=0.56)$. Increases in defecation frequency (3 patients) and abdominal cramp (2 patients) were the most common side effects that the participants noted.

\section{Discussion}

Rose oil soft capsules significantly decreased partial mayo clinic and IBDQ-9 scores at the end of the study, though this change was also observed in the placebo group. The fall detected in calprotectin levels -an objective sign of 


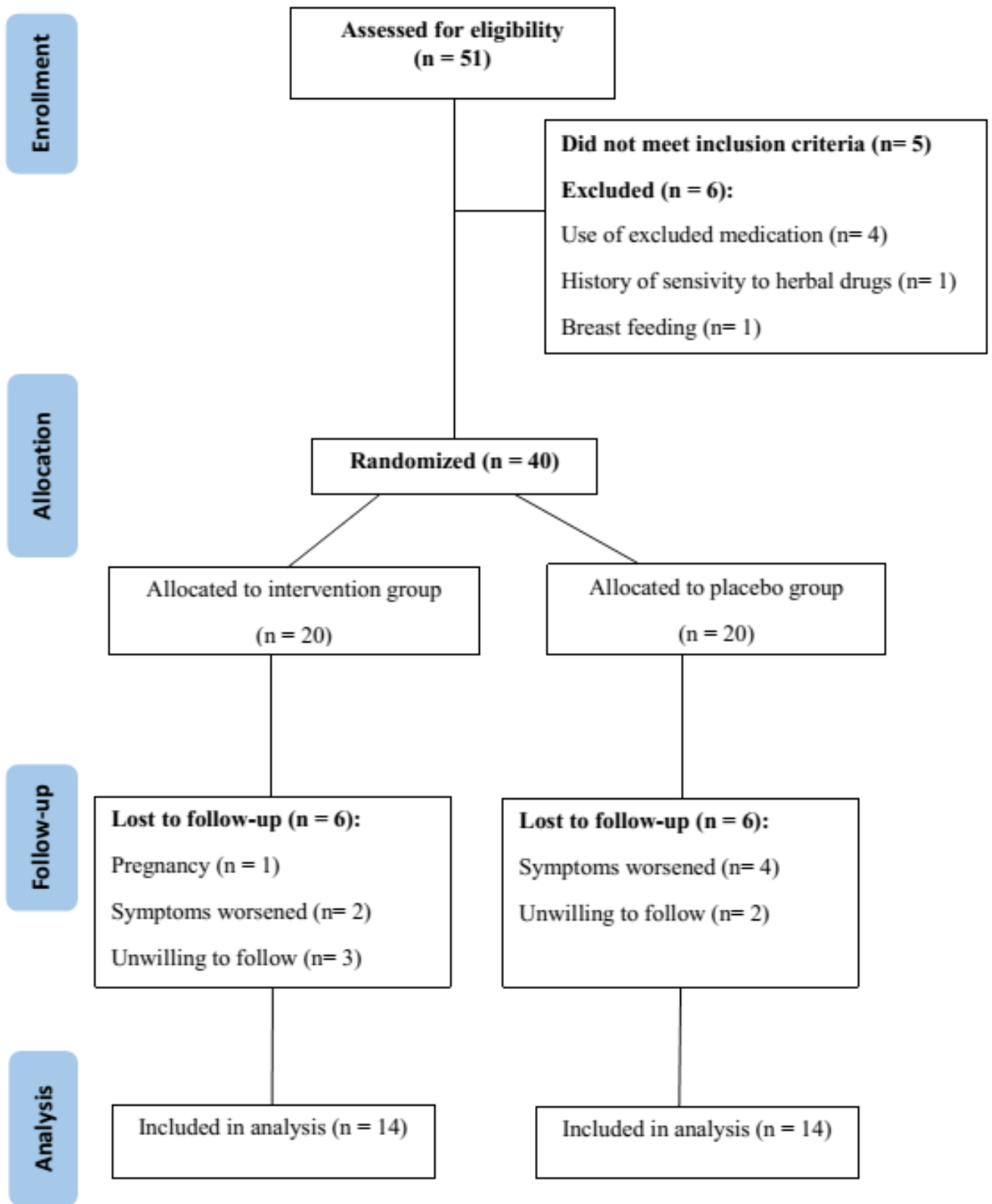

figure 1. Consort flow diagram of the trial

ameliorating inflammation mediators - was not statistically significant. Flowers and petals of damask rose contain flavonoids, polyphenols, and vitamin $\mathrm{C}$, and it has been reported that flavonoids and polyphenols have anti-inflammatory effects, especially in the gastrointestinal tract [20]. Anti-inflammatory properties of flavonoids have been demonstrated in previous experimental studies. Flavonoids have also shown anti-ulcer effects [21, 22]. Oral administration of geraniol (one of the components of rose oil) has been a potential therapeutic agent for experimental colitis in mice and significantly reduced colitis clinical signs and pro-inflammatory indices [14]. Another animal study revealed that geraniol with its antioxidant and anti-inflammatory properties improved experimental colitis in rats [13]. Also, the sesame oil- which is used as a carrier for preparing rose oil- is effective in the animal model of colitis and inflammation. Oral administration of sesame oil accelerated the healing of an inflamed colon in rats [23]. Another study indicated that sesamol (one of the active constituents of sesame oil) has mucosal protective effects for IBD in rats [24]. 
Table 1. The Baseline Demographic and Primary Characteristics in Both Intervention Groups

\begin{tabular}{|c|c|c|c|c|}
\hline \multirow{2}{*}{ Variables } & \multirow{2}{*}{ Total } & \multicolumn{2}{|c|}{ Group } & \multirow{2}{*}{ P-value } \\
\hline & & Rose $(n=14)$ & Placebo $(n=14)$ & \\
\hline \multicolumn{4}{|l|}{ Age (years) } & \multirow{2}{*}{$0.499 \dagger$} \\
\hline Mean \pm SD & $41 \pm 10$ & $40 \pm 10$ & $42 \pm 11$ & \\
\hline \multicolumn{5}{|l|}{ Gender } \\
\hline Female & $13(46.4 \%)$ & $5(35.7 \%)$ & $8(57.1 \%)$ & \multirow{2}{*}{$0.225 *$} \\
\hline Male & $15(53.6 \%)$ & $9(64.3 \%)$ & $6(42.9 \%)$ & \\
\hline \multicolumn{5}{|l|}{ Duration of UC } \\
\hline$<5$ years & $9(32.1 \%)$ & $6(42.9 \%)$ & $3(21.4 \%)$ & \multirow{3}{*}{$0.376 \div$} \\
\hline $5 \mathrm{y} \leq$ and $<10 \mathrm{y}$ & $8(28.6 \%)$ & $2(14.2 \%)$ & $6(42.9 \%)$ & \\
\hline$\geq 10$ years & $11(39.3 \%)$ & $6(42.9 \%)$ & $5(35.7 \%)$ & \\
\hline \multicolumn{5}{|c|}{ Hospital Admission (n) } \\
\hline Yes & $9(32.1 \%)$ & $4(28.6 \%)$ & $5(35.7 \%)$ & \multirow{2}{*}{$0.5 *$} \\
\hline No & $19(67.9 \%)$ & $10(71.4 \%)$ & $9(64.3 \%)$ & \\
\hline
\end{tabular}

Severity of diagnosis

$$
\begin{gathered}
\text { Proctitis } \\
\text { Left. sided Colitis } \\
\text { Extended Colitis } \\
\text { Pan-Colitis } \\
\text { Stool frequency } \\
\text { Normal } \\
\text { 1-2 times more } \\
\text { 3-4 times more } \\
5 \text { times more }
\end{gathered}
$$

\section{Rectal bleeding}

$$
\begin{gathered}
\text { Normal } \\
\text { Bleeding }<1-2 \\
\text { Bleeding }>1-2 \\
\text { Always Bleeding }
\end{gathered}
$$

$$
\begin{gathered}
5(17.9 \%) \\
8(28.6 \%) \\
3(10.7 \%) \\
12(42.8 \%)
\end{gathered}
$$

$2(14.3 \%)$

$4(28.6 \%)$

$2(14.3 \%)$

$6(42.8 \%)$

$1(3.6 \%)$

$14(50.0 \%)$

$9(32.1 \%)$

$4(14.3 \%)$

$$
\begin{gathered}
19(67.8 \%) \\
5(17.9 \%) \\
3(10.7 \%) \\
1(3.6 \%)
\end{gathered}
$$

$0(0.0 \%)$

$7(50.0 \%)$

$5(35.7 \%)$

$2(14.3 \%)$

$$
\begin{gathered}
9(64.3 \%) \\
2(14.3 \%) \\
2(14.3 \%) \\
1(7.1 \%)
\end{gathered}
$$

$10(17.9 \%)$

$13(28.6 \%)$

$7(50.0 \%)$

$5(35.7 \%)$

$2(14.3 \%)$

$$
\begin{aligned}
& 3(21.4 \%) \\
& 8(57.2 \%)
\end{aligned}
$$$$
3(21.4 \%)
$$

$10(71.5 \%)$

$3(21.4 \%)$

$1(7.1 \%)$

$0(0.0 \%)$

0.635 +

$41.6 \pm 9.5$

$44.6 \pm 9.4$

0.408 †

Partial Mayo Clinic Score

Mean \pm SD
$\uparrow$ based on t-test
$*$ Based on Chi-Square test
+ Based on Mann-Whitney test.

\section{(4).}

$3.86 \pm 1.46$

$0.921 \dagger$

\footnotetext{
* Based on Chi-Square test

$\$$ Based on Mann-Whitney test.
} 
Table 2. The Differences of Primary Outcome Measures Between 2 Intervention Groups

\begin{tabular}{|c|c|c|c|c|c|c|c|}
\hline \multirow{2}{*}{ Parameters } & \multirow{2}{*}{ Time } & \multicolumn{2}{|c|}{ Group } & \multirow{2}{*}{ Diff. } & \multicolumn{2}{|c|}{ 95\% CI } & \multirow{2}{*}{$\begin{array}{c}\text { P- } \\
\text { value }\end{array}$} \\
\hline & & Rose $(n=14)$ & $\begin{array}{c}\text { Placebo } \\
(n=14)\end{array}$ & & Lower & Upper & \\
\hline \multirow{5}{*}{$\begin{array}{c}\text { Stool } \\
\text { frequency }\end{array}$} & Baseline & $1.64 \pm 0.75$ & $1.50 \pm 0.86$ & 0.14 & -0.48 & 0.77 & $0.667+$ \\
\hline & After $1 \mathrm{M}$ & $1.14 \pm 0.54$ & $1.0 \pm 0.56$ & 0.14 & -0.28 & 0.57 & 0.603 \\
\hline & P-Withine & 0.020 & 0.035 & & & & \\
\hline & After $2 \mathrm{M}$ & $0.86 \pm 0.77$ & $0.93 \pm 0.62$ & -0.07 & -0.61 & 0.47 & 0.769 \\
\hline & P-Withine & 0.021 & 0.074 & & & & \\
\hline \multirow{5}{*}{$\begin{array}{c}\text { Rectal } \\
\text { bleeding }\end{array}$} & Baseline & $0.64 \pm 1.01$ & $0.36 \pm 0.63$ & 0.28 & -0.37 & 0.94 & $0.635+$ \\
\hline & After $1 \mathrm{M}$ & $0.29 \pm 0.61$ & $0.14 \pm 0.54$ & 0.14 & -0.30 & 0.59 & 0.571 \\
\hline & P-Within & 0.102 & 0.257 & & & & \\
\hline & After $2 \mathrm{M}$ & $0.14 \pm 0.36$ & $0.07 \pm 0.27$ & 0.07 & -0.18 & 0.32 & 0.769 \\
\hline & P-Withine & 0.066 & 0.102 & & & & \\
\hline \multirow{5}{*}{$\begin{array}{c}\text { Physician } \\
\text { global } \\
\text { assessment }\end{array}$} & Baseline & $1.64 \pm 0.75$ & $2.0 \pm 0.68$ & -0.36 & -0.91 & 0.2 & $0.210 \div$ \\
\hline & After $1 \mathrm{M}$ & $1.36 \pm 0.63$ & $1.43 \pm 0.76$ & -0.07 & -0.61 & 0.47 & 0.910 \\
\hline & P-Within & 0.046 & 0.011 & & & & \\
\hline & After $2 \mathrm{M}$ & $1.14 \pm 0.77$ & $1.14 \pm 0.86$ & 0.0 & -0.64 & 0.64 & 1 \\
\hline & P-Withine & 0.068 & 0.014 & & & & \\
\hline \multirow{3}{*}{ IBD-Q9 } & Baseline & $41.6 \pm 9.5$ & $44.6 \pm 9.4$ & -3.0 & -10.3 & 4.3 & $0.408 \dagger$ \\
\hline & After $2 \mathrm{M}$ & $47.5 \pm 8.3$ & $48.9 \pm 6.5$ & -1.4 & -7.2 & 4.4 & 0.617 \\
\hline & P-Within & 0.03 & 0.012 & & & & \\
\hline \multirow{5}{*}{$\begin{array}{c}\text { Partial } \\
\text { Mayo Clinic } \\
\text { Score }\end{array}$} & Baseline & $3.93 \pm 2.24$ & $3.86 \pm 1.46$ & 0.07 & -1.40 & 1.54 & $0.921 \dagger$ \\
\hline & After $1 \mathrm{M}$ & $2.79 \pm 1.37$ & $2.57 \pm 1.34$ & 0.21 & -0.84 & 1.27 & 0.679 \\
\hline & P-Within & 0.014 & 0.013 & & & & \\
\hline & After $2 \mathrm{M}$ & $2.14 \pm 1.61$ & $2.14 \pm 1.46$ & 0.0 & -1.19 & 1.19 & 1 \\
\hline & P-Within & 0.022 & 0.014 & & & & \\
\hline
\end{tabular}

CI: Confidence Interval, Pre: at the starting of the study, after 1M: 1 month after using drugs and after 2 M: 2 months after starting the study (end point of the study).

$\dagger$ Based on t-test

$\$$ Based on Mann-Whitney test

I Based on Paired t-test

. Based on Wilcoxon Signed rank test 
Table 3. The Comparison of the Patients' Calprotectin Level in Intervention Groups

\begin{tabular}{cccc}
\hline Groups & $\begin{array}{c}\text { Before intervention } \\
(\text { Mean } \pm \text { SD) }\end{array}$ & $\begin{array}{c}\text { After intervention } \\
(\text { Mean } \pm \text { SD) }\end{array}$ & P-value $\dagger$ \\
\hline Rose oil group & $64.21 \pm 93.47$ & $34.75 \pm 89.45$ & 0.229 \\
Controlled group & $67.56 \pm 138.19$ & $33.45 \pm 2.72$ & 0.122 \\
\hline
\end{tabular}

$\dagger$ based on paired t-test

Table 4. The Comparison of the Patients' Satisfaction between Intervention Groups After Two Months

\begin{tabular}{|c|c|c|c|c|c|c|c|}
\hline \multirow[b]{2}{*}{ Parameter } & \multirow[b]{2}{*}{ Time } & \multicolumn{2}{|c|}{ Groups } & \multirow[b]{2}{*}{ Diff } & \multicolumn{2}{|c|}{$95 \%$ CI } & \multirow[b]{2}{*}{ P-value $\dagger$} \\
\hline & & Rose oil $(n=14)$ & $\begin{array}{c}\text { Placebo } \\
(n=14)\end{array}$ & & Lower & Upper & \\
\hline VAS & $\begin{array}{l}\text { At the } \\
\text { end of } \\
\text { study }\end{array}$ & $7.46 \pm 2.33$ & $6.79 \pm 2.01$ & 0.67 & -1.05 & 2.39 & 0.426 \\
\hline
\end{tabular}

CI: Confidence Interval

$\dagger$ Based on t-test

These findings may justify the promising effect of rose oil soft capsules for relieving partial Mayo clinic and IBDQ-9 scores at the end of the study. In spite of large administration of herbal remedies for curing UC and its symptoms, shortage of high-quality design clinical studies proving the efficacy and safety of these products is obvious $[25,26]$. In a multicenter placebo-controlled study on Andrographis paniculata extract at an oral dose of 1800 mg, a relatively acceptable clinical response was reported by Sandborn et al. [27]. The authors prescribed this product for eight weeks to mild to moderate UC patients in five different countries [27]. Our findings are in line with this study, but it should be noticed that this herb is not an endemic plant, which was imported from neighboring countries years ago [28]. Langmead et al. [29] revealed the positive effect of oral consumption of aloe vera gel in $44 \mathrm{UC}$ patients. The results were similar to ours in term of clinical outcomes and safety profile considering their shorter treatment period (four weeks), though they explored histological aspects too [29]. The difference between active and placebo groups was not statistically significant in both investigations. Gong et al. [30] examined a traditional Chinese medicine formula (Fufangkushen colon-coated capsules) in comparison to mesalazine for eight weeks. The results indicated that this remedy could be an effective and safe option, though the control group revealed the same response [30]. The results are in accordance with ours in the abovementioned aspects, although the sample size was larger in the Chinese group (320 vs. 39 patients). Both rose oil soft capsule and placebo (liquid paraffin) groups showed promising results in this survey. It may be attributed to the effects of preferred placebo. As the safety of the placebo was so imperative for the authors, the item selected for the control group was not very optimal. Paraffin (mineral oil) has shown partially accepted effects for increased bowel movements in children [31]. So, it may affect the inflammatory features of the disease, causing fewer symptoms in UC patients. Even though we preferred a choice of treatment with an inclusive analysis profile (rose oil capsule), we did not recognize the definite active ingredient(s) to explore the mechanism of action in 
this piece of research -a common weakness in such trials $[26,32]$. Consideration of holistic approach in traditional and complementary/alternative medical systems could well explain this shortcoming. The synergistic effect of bioactive substances is also a superior rationale for convincing the pessimistic researchers [33]. Alternatively, finding the main active ingredient was not set as an outcome for our investigation and could be scrutinized in future pharmacological studies. We used calprotectin for an objective examination of our hypothesis that showed a descending trend in the course of our study. However, this was not statistically significant while observing inner and between-group differences. Notably, the power of these data was more than $97 \%$. This shows that such a small sample size is undoubtedly satisfactory for this item. At present, traditional/complementary and alternative modalities have are gaining popularity in different medical fields. Use of these options for the treatment of UC, as adjunctive therapy beside conventional medications, and as a holistic approach in the therapeutic process is very common nowadays [32]. Persian medicine (PM) -sometimes called Unani medicine - one of the most acknowledged medical systems from the medieval period, has been under the practice in Iran and many countries for a long history [34]. Based on PM perspective, temperaments and humors should be kept in mind while treating the patients, thus, considering these factors rose oil could act as a tonic agent in large intestine and could throw out the waste material including excess unbalanced humors from the site [11, 35]. On the other hand, rose oil could play a role as a soothing agent, and consequently, it could adequately decrease the symptoms of local inflammation [9]. Although anti-inflammatory and ulcer-healing effects of $R$. damascena have been demonstrated in several studies, and the effects of some components of this plant and the rose oil on experimental colitis have been shown in some animal studies $[14,36]$, to the best our knowledge, there is no clinical trial study on the efficacy of rose oil in treating UC. Acceptable safety profile can be mentioned as an advantage of the current study, as there were no harmful or life-threatening side effects during the treat- ment period. Gastrointestinal symptoms were the chief complaint in these groups causing them to voluntary quit the study. The participants were also satisfied with the suggested medicines in both groups, though this item did not show any significant difference. Our study had some limitations; relatively small sample size and short-time follow-up were the main limitations of our study. A glance at the results of study reveals that the powers of these data are low indeed. This means if the sample size of the study grows, it may result in significant differences between the two products. Use of an affordable product which has gone through all legal and scientific procedures in terms of quality control profile from relevant authorities was an important superiority in this study.

\section{Conclusion}

As mentioned previously, both rose oil and placebo may be effective for UC. However, comparing the effects of these two products, no significant difference was observed between the two study groups at the end of the study. This study was a pilot study with a small research population. Experiments with larger sample populations through multicenter clinical investigations with follow-up observation should be conducted to validate the findings of this study.

\section{Acknowledgment}

This work was supported through a collaborative project between Shiraz University of Medical Sciences and Barij Essence Pharmaceutical Company (grant number: BE-113 ). This study was a part of the Ph.D. dissertation of Dr. Ali Tavakoli for the Iranian Board of Traditional Medicine (94-01-64-10329). We would also like to extend our thanks to Dr. Hossein Molavi for his support in statistical analysis.

\section{Conflict of interest}

The authors declare that there is no conflict of interest. Leila Mohammad Taghizadeh is an employee of Barij Essence Pharmaceutical Company. 
1. Ungaro R, Mehandru S, Allen PB, PeyrinBiroulet L, Colombel JF. Ulcerative colitis. Lancet 2017; 389(10080): 1756-70.

2. Peyrin-Biroulet L, Sandborn W, Sands BE, Reinisch W, Bemelman W, Bryant RV et al. Selecting Therapeutic Targets in Inflammatory Bowel Disease (STRIDE): Determining Therapeutic Goals for Treatto-Target. Am J Gastroenterol 2015; 110(9): 1324-38.

3. Gibson PR, Vaizey C, Black CM, Nicholls R, Weston AR, Bampton P et al. Relationship between disease severity and quality of life and assessment of health care utilization and cost for ulcerative colitis in Australia: A cross-sectional, observational study. J Crohns Colitis 2014; 8(7): 598-606.

4. Baumgart DC, Sandborn WJ. Inflammatory bowel disease: clinical aspects and established and evolving therapies. Lancet 2007; 369(9573): 1641-57.

5. Kornbluth A, Sachar DB. Ulcerative colitis practice guidelines in adults: American College Of Gastroenterology, Practice Parameters Committee. Am J Gastroenterol 2010; 105(3): 501-23; quiz 24.

6. Ford AC, Sandborn WJ, Khan KJ, Hanauer SB, Talley NJ, Moayyedi P. Efficacy of biological therapies in inflammatory bowel disease: systematic review and meta-analysis. Am J Gastroenterol 2011; 106(4): 644-59, quiz 60.

7. Jagtap AG, Shirke SS, Phadke AS. Effect of polyherbal formulation on experimental models of inflammatory bowel diseases. $\mathrm{J}$ Ethnopharmacol 2004; 90(2-3): 195-204.

8. Razi MZ. Al-Havi Fi Al-Tibb (Arabic). Beirut: Dar Al Kotob Al-islmiyah; 2000.

9. Aghili Shirazi MH. Qarabadin-e Kabir (Great Pharmacopoeia). Tehran: Institute of Meical History, Islamic Medicine and Complementary Medicine; Iran Medical University; 1970.

10. Mahboubi M. Rosa damascena as holy ancient herb with novel applications. J Tradit Complement Med 2016; 6(1): 10-6.

11. Avicenna. Al-Qanun fi al-Tibb (The Canon of Medicine). Tehran: Soroush Press; 2005.

12. Loghmani H, Sabzi O, Safari J. Essential oil composition of Rosa damascena Mill cultivated in central Iran. Scientia Iranica 2007; 14: 316-9.
13. Soubh AA, Abdallah DM, El-Abhar HS. Geraniol ameliorates TNBS-induced colitis: Involvement of Wnt/beta-catenin, p38MAPK, NFkappaB, and PPARgamma signaling pathways. Life Sci 2015; 136: 14250.

14. Medicherla K, Sahu BD, Kuncha M, Kumar JM, Sudhakar G, Sistla R. Oral administration of geraniol ameliorates acute experimental murine colitis by inhibiting pro-inflammatory cytokines and NF-kappaB signaling. Food Funct 2015; 6(9): 2984-95.

15. Cohen BL, Zoega H, Shah SA, Leleiko N, Lidofsky S, Bright R et al. Fatigue is highly associated with poor health-related quality of life, disability and depression in newlydiagnosed patients with inflammatory bowel disease, independent of disease activity. Aliment Pharmacol Ther 2014; 39(8): 81122.

16. Habtemariam S, Belai A. Natural Therapies of the Inflammatory Bowel Disease: The Case of Rutin and its Aglycone, Quercetin. Mini Rev Med Chem 2018; 18(3): 234-43.

17. Lewis JD, Chuai S, Nessel L, Lichtenstein GR, Aberra FN, Ellenberg JH. Use of the noninvasive components of the Mayo score to assess clinical response in ulcerative colitis. Inflamm Bowel Dis 2008; 14(12): 1660-6.

18. Bijari B, Soltani B. Validation of the Persian Version of Inflammatory Bowel Disease Questionnaire in Patients Who Referred to Clinics and Hospitals of Birjand University of Medical Sciences, Iran. Ann Colorectal Res 2017; 5(1): e46367.

19. Hosseini SV, Jafari P, Taghavi SA, Safarpour AR, Rezaianzadeh A, Moini M et al. Fecal Calprotectin is an Accurate Tool and Correlated to Seo Index in Prediction of Relapse in Iranian Patients With Ulcerative Colitis. Iran Red Crescent Med J 2015; 17(2): e22796.

20. Gonzalez R, Ballester I, Lopez-Posadas R, Suarez MD, Zarzuelo A, Martinez-Augustin $\mathrm{O}$ et al. Effects of flavonoids and other polyphenols on inflammation. Crit Rev Food Sci Nutr 2011; 51(4): 331-62.

21. Garcia-Lafuente A, Guillamon E, Villares A, Rostagno MA, Martinez JA. Flavonoids as anti-inflammatory agents: implications in cancer and cardiovascular disease. Inflamm 
Res 2009; 58(9): 537-52.

22. Yassa N, Masoomi F, Rohani Rankouhi S, Hadjiakhoondi A. Chemical Composition and Antioxidant Activity of the Extract and Essential oil of Rosa damascena from Iran, Population of Guilan. Daru 2009; 17(3): 175-80.

23. Periasamy S, Hsu DZ, Chandrasekaran VR, Liu MY. Sesame oil accelerates healing of 2,4,6-trinitrobenzenesulfonic acid-induced acute colitis by attenuating inflammation and fibrosis. JPEN J Parenter Enteral Nutr 2013; 37(5): 674-82.

24. Kondamudi PK, Kovelamudi H, Mathew G, Nayak PG, Rao MC, Shenoy RR. Investigation of sesamol on myeloperoxidase and colon morphology in acetic acid-induced inflammatory bowel disorder in albino rats. Scientific World J 2014; 2014: 802701.

25. Sebepos-Rogers GM, Rampton DS. Herbs and Inflammatory Bowel Disease. Gastroenterol Clin North Am 2017; 46(4): 809-24.

26. Langmead L, Rampton DS. Review article: complementary and alternative therapies for inflammatory bowel disease. Aliment Pharmacol Ther 2006; 23(3): 341-9.

27. Sandborn WJ, Targan SR, Byers VS, Rutty $\mathrm{DA}, \mathrm{Mu} \mathrm{H}$, Zhang X et al. Andrographis paniculata extract (HMPL-004) for active ulcerative colitis. Am J Gastroenterol 2013; 108(1): 90-8.

28. Valdiani A, Kadir MA, Tan SG, Talei D, Abdullah MP, Nikzad S. Nain-e Havandi Andrographis paniculata present yesterday, absent today: a plenary review on underutilized herb of Iran's pharmaceutical plants. Mol Biol Rep 2012; 39(5): 5409-24.

29. Langmead L, Feakins RM, Goldthorpe S, Holt H, Tsironi E, De Silva A et al. Randomized, double-blind, placebocontrolled trial of oral aloe vera gel for active ulcerative colitis. Aliment Pharmacol Ther 2004; 19(7): 739-47.
30. Gong Y, Zha Q, Li L, Liu Y, Yang B, Liu L et al. Efficacy and safety of Fufangkushen colon-coated capsule in the treatment of ulcerative colitis compared with mesalazine: a double-blinded and randomized study. $\mathrm{J}$ Ethnopharmacol 2012; 141(2): 592-8.

31. Gordon M, MacDonald JK, Parker CE, Akobeng AK, Thomas AG. Osmotic and stimulant laxatives for the management of childhood constipation. Cochrane Database Syst Rev 2016; (8): Cd009118.

32. Zezos P, Nguyen GC. Use of Complementary and Alternative Medicine in Inflammatory Bowel Disease Around the World. Gastroenterol Clin North Am 2017; 46(4): 679-88.

33. Pasalar M, Choopani R, Mosaddegh M, Kamalinejad M, Mohagheghzadeh A, Fattahi MR et al. Efficacy and safety of jollab to treat functional dyspepsia: a randomized placebocontrolled clinical trial. Explore (NY) 2015; 11(3): 199-207.

34. Sedigh-Rahimabadi M, Fani M, RostamiChijan M, Zarshenas MM, Shams M. A Traditional Mouthwash (Punica granatum var pleniflora) for Controlling Gingivitis of Diabetic Patients: A Double-Blind Randomized Controlled Clinical Trial. J Evid Based Complementary Altern Med 2017; 22(1): 59-67.

35. Mosaffa-Jahromi M, Pasalar M, Afsharypuor S, Choopani R, Mosaddegh M, Kamalinejad $\mathrm{M}$ et al. Preventive care for gastrointestinal disorders; role of herbal medicines in traditional persian medicine. Jundishapur J Nat Pharm Prod 2015; 10(4).

36. Hajhashemi V, Ghannadi A, Hajiloo M. Analgesic and Anti-inflammatory Effects of Rosa damascena Hydroalcoholic Extract and its Essential Oil in Animal Models. Iran J Pharm Res 2010; 9(2): 163-8. 